\title{
The effect of application of a Soton ureteroscope on infection after flexible ureteroscopy lithotripsy
}

\author{
Xiaobo Zhang, Zhenyu Liu, Xiong Chen, Dongjie Li, Zhiming Yang, Jie Gu, Sheng Hu, Yuxin Li \\ Xiangya Hospital Central South University, China
}

Videosurgery Miniinv 2022; 17 (1): 232-239

DOI: https://doi.org/10.5114/wiitm.2021.107763

\begin{abstract}
Introduction: Postoperative infection is still one of the most common complications following flexible ureteroscopy lithotripsy (FURL). However, whether a combination of negative pressure ureteroscopy (and Soton ureteroscopy) is superior to FURL in lithotripsy with regard to intraoperative pressure and possibly the incidence of postoperative infection remains to be validated.

Aim: To explore the effect of a Soton ureteroscope on infection following flexible ureteroscope lithotripsy.

Material and methods: Sixty patients with kidney stones were randomly divided equally into study and control groups. The operation duration, stone-free rate, postoperative blood routine, procalcitonin, C-reactive protein, and other data between the two groups were then analysed and compared.

Results: There were no statistically significant differences between the study group and the control group regarding the average operation time and the average number of hospitalization days. The mean stone-free rate 1 week after surgery and mean VAS pain score 1 day after surgery for the study group and the control group were 91.3\% and 0.27 vs. $76.9 \%$ and 0.61 , respectively. Notably, the average body temperature after the first day of the operation was $36.4^{\circ} \mathrm{C}$ in the study group and $36.7^{\circ} \mathrm{C}$ in the control group. More importantly, concerning postoperative infection index, white blood cells (WBCS), percentage of neutrophils, C-reactive protein, and procalcitonin were all lower in the study group than in the control group.

Conclusions: Compared with flexible ureteroscopy alone, combined use of Soton ureteroscopy is associated with fewer substantially infection following lithotripsy.
\end{abstract}

Key words: renal stones, Soton ureteroscopy, flexible ureteroscopy lithotripsy, infection.

\section{Introduction}

Kidney stones are amongst the most prevalent diseases in urology. Severe cases might lead to kidney damage, affecting the kidney's physiological function. At present, kidney stones are mainly treated by surgery, and minimally invasive treatment is the current trend. Currently, the 3 major lithotripsy methods are extracorporeal shock wave lithotripsy (ESWL), percutaneous nephrolithotripsy (PNL) and ureteroscopic lithotripsy (URL) [1, 2].
Recently, flexible ureteroscopy lithotripsy (FURL) has also been widely used in the clinical treatment of kidney stones [3, 4]. Even with the introduction and application of various new surgical techniques, the incidence of complications after lithotripsy is still relatively high, although the operation effect has significantly improved $[3,5]$. Postoperative infection is one of the most frequently reported complications, and in severe cases, systemic inflammatory response syndrome, or even septic shock, seriously threatens the life of patients $[6,7]$. Hence, the oc- 
currence and clinical implication of infection after lithotripsy cannot be ignored [8].

Significantly, using flexible ureteroscopy to treat kidney stones has shown favourable results over the years. Nonetheless, the continuous filling and fluid flushing required during the lithotriptic process to maintain a clear field of vision has remained the downside to its use. The continuous filling and fluid flushing leads to possible absorption of bacteria and endotoxin into the bloodstream, increasing the risk of postoperative infection [9].

Thus, the use of Soton ureteroscopy (combined negative pressure ureteroscopy) has the characteristics of a relatively non-invasive, safe, and effective operation via the natural cavity. During lithotripsy, negative-pressure suction is used to remove stone fragments and powder, reducing intraoperative pressure and maintain a clear intraoperative vision.

\section{Aim}

We aimed to compare postoperative infection among renal calculi patients who underwent Soton ureteroscopy in combination with flexible ureteroscopy or flexible ureteroscopy alone.

A

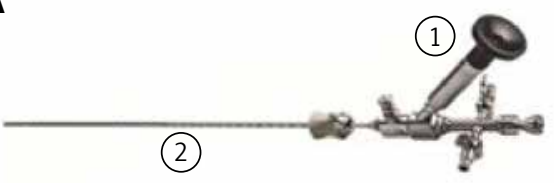

D

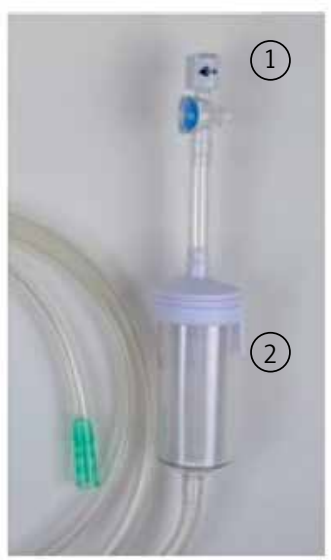

\section{Material and methods}

\section{Patients}

We enrolled 60 patients with renal stones, who underwent treatment from April 2018 to December 2018 at our hospital. Only patients (i) between 18 and 75 years of age and stable for surgery, (ii) with renal stones measuring between 1.5 and $2.5 \mathrm{~cm}$, (iii) and willingly consenting for this study were included.

Sixty patients who met the above criteria were first randomly divided into 2 equal groups (study and control groups; $n=30$ each). The study group underwent a combination of Soton ureteroscopy and flexible ureteroscopy (Olympus), whereas the control group underwent flexible ureteroscopy alone (Olympus).

The study was approved by both the ethics committee and the review board of our hospital. All procedures involving human participants were performed according to the institutional and or national research committee's ethical standards.

\section{Device of Soton}

The Soton ureteroscope comprises 5 main components, including a standard ureteroscope (Photo 1 A),

B

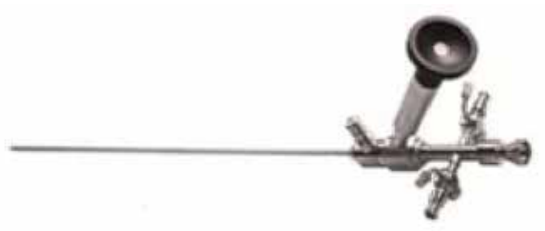

C
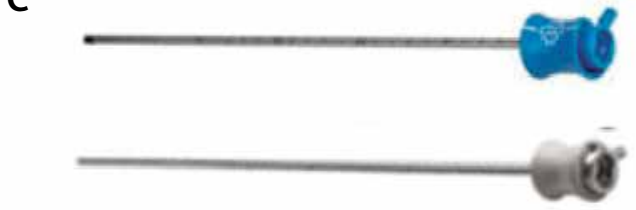

$\mathrm{E}$

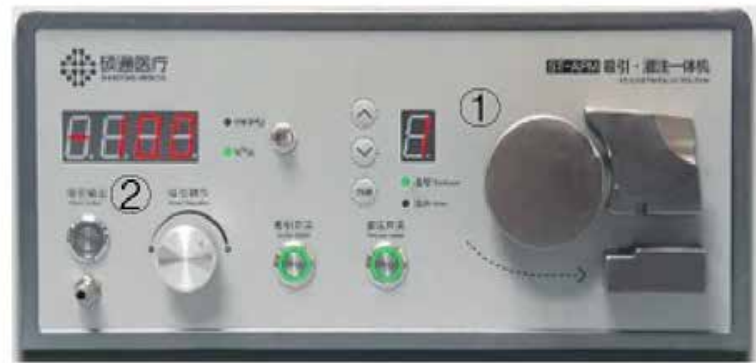

Photo 1. Soton ureteroscope components. A - 1) Standard ureteroscope, 2 rigid ureteral access sheath. B - Console ureteroscope. C - Rigid ureteral access sheath. D - 1) Switch for adjusting negative pressure, 2) container for stone dust collection. E - Irrigation and suctioning platform 


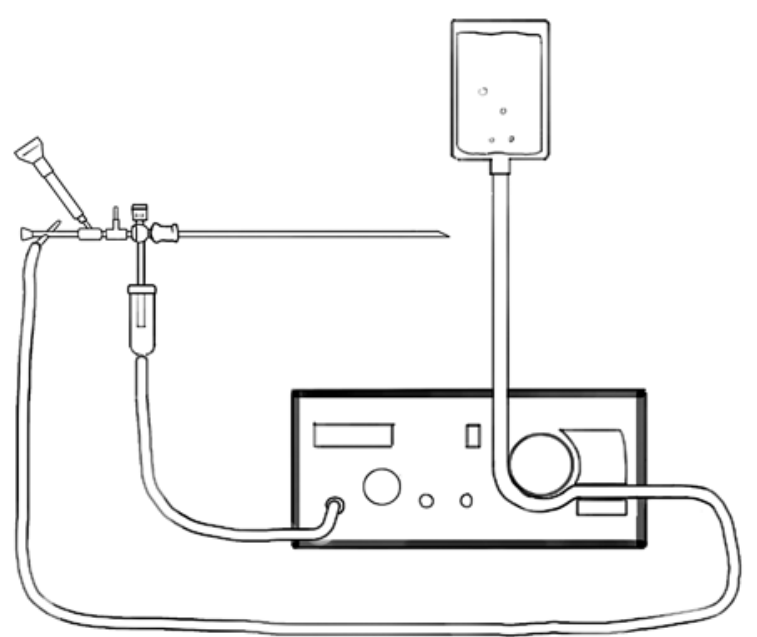

Figure 1. Assembly diagram. A simple schematic of the Soton ureteroscope components during the operation

a console ureteroscope (Photo $1 \mathrm{~B}$ ), a rigid ureteral access sheath (Photo $1 \mathrm{C}$ ), a switch for adjusting the negative pressure (Photo $1 \mathrm{D})$, and an irrigation and suctioning platform (Photo $1 \mathrm{E}$ ).

The standard ureteroscope is $45 \mathrm{~cm}$ in length, with an outer diameter of 7.5 (tip)/9.8 (shaft) F. The console ureteroscope is longer but smaller than the standard ureteroscope, with a length of $46 \mathrm{~cm}$, an outer diameter of 4.0/6.0 F. The outer diameter of the rigid ureteral access sheath is $12.5 / 13.5 \mathrm{~F}$. During the operation, the surgeon can manually adjust the negative pressure by adjusting the switch, and the lower end of the adjusting switch is connected to a container for stone dust collection. The irrigation and suctioning platform can be preset to perfusion and suctioning modes. Perfusion has continuous or pulsed modes, which can be easily switched. The perfusion flow speed ranged from 60 to $610 \mathrm{ml} / \mathrm{min}$, and the setting range of suction negative pressure is -25 to $-4 \mathrm{kPa}$. Figure 1 is a simple schematic of the Soton ureteroscope components during the operation.

\section{Methods}

Respective surgeries were approved after sufficient assessment of the patients. Vital signs were continually monitored throughout the operation. All patients were administered with general anaesthesia before the operation. We used the holmium laser system, perfusion flush, and a negative-pressure suction system all from the same manufacturer. The procedures were also performed by the same group of urologists. Patients were administered with appropriate prophylactic antibiotics both preoperatively and intraoperatively. The surgery was performed in the lithotomy position.

The ureter of the operative side of patients in the control group was assessed using an 8.0 9.5 F ureteroscope, with a Cook smooth guidewire placed in the renal pelvis. Thereafter, an F12 Cook ureteral access sheath and the Olympus electronic flexible ureteroscope were inserted into the ureter before examining all the renal calyces in turn. The kidney stones were fragmented using a Holmium laser. The larger stones were removed using a stone basket. Each renal calyx was examined again after surgery. The whole ureter was also examined while removing the ureteroscope. A ureteral stent was fixed in the ureter.

For the study group, we combined the standard ureteroscope (F7.5) and a rigid ureteral access sheath (F13.5). Here, an F4 guidewire was first advanced into the upper ureteral segment or the renal pelvis. The standard ureteroscope was then removed but the sheath remained. The rigid sheath with a negative-pressure suction device and the perfusion suction devices were then connected simultaneously to establish a working channel.

The ureteroscope was then passed through the working channel to the kidneys. The fragmented stones and powder were removed through negative-pressure suction during the lithotripsy. The strength of the negative pressure was regulated to control the suction rate of fluid and clastic stones. After removing the ureteroscope, a flexible ureteroscope was inserted into the rigid ureteral access sheath to reach the stones in the lower renal calyx.

Then after stones were broken into fragments, water pressure was used to wash them out or they were placed in the renal pelvis or renal calyx where they could be dealt with by a stone basket, then the previous ureteroscope was exchanged to break the stones. Perfusion and negative-pressure suction were stopped after careful assessment of the renal pelvis and calyxes. The renal pelvis and calyxes were carefully examined for any stone remnants before stopping the negative-pressure suction. Then the ureteral access sheath and the conventional ureteroscope were tied together and removed while simultaneously examining the renal and ureter. Finally, a ureteral stent was fixed to the ureter.

The general characteristics of all patients were captured. In addition, blood and urine tests were 
performed during the perioperative and postoperative periods. Blood routine tests were performed 1 day postoperatively, whereas procalcitonin (PCT) and C-reactive protein (CRP) serum levels were re-examined 24-hours after surgery [10-13]. A plain abdominal X-ray was performed 1 week after the surgery to calculate the stone-free rate (SFR).

These data were statistically analysed to compare the degree and rate of postoperative infection among the 2 groups of patients. The present study's primary outcome was postoperative infections defined as fever $\left(\geq 38^{\circ} \mathrm{C}\right)$, pyuria ( $\geq 10$ WBCs per high power field), systemic inflammatory response syndrome (SIRS), or sepsis.

\section{Statistical analysis}

The operative time (calculated from the establishment of the lithotripsy passage to the successful placement of ureteral stent), SFR, visual analogue score (VAS), average duration of hospital stay, perioperative and postoperative condition, and incidences of infection were all compared between the 2 groups. Data were analysed using Statistical Package for IBM SPSS Statistics 22.

Continuous variables were expressed as mean (standard deviation - SD). Differences between groups were compared using independent $t$-tests. Categorical variables were compared using $\chi^{2}$ tests. $P$-value $<0.05$ was considered statistically significant.

\section{Results}

There was no significant statistical difference in basic data among patients between the 2 groups $(p>0.05)$. In the study group, 1 patient had a positive "+" urine sign in the qualitative measurement of white blood cells (WBC) count, although the urine culture was negative. In contrast, 2 patients in the control group had a positive "+++" urine sign in the qualitative measurement of WBC count in the urine with a positive culture. These patients were put on antibiotic treatment until turning negative for both blood culture and qualitative urine WBC count. Subsequently, lithotripsy was performed. Detailed results are shown in Table I.

Intraoperative and postoperative patients: Intraoperative blood loss, operation time, body temperature after the first operative day, VAS pain score, postoperative catheter indwelling time, hospital- ization days, and other relevant data between the 2 groups were statistically analysed. All patients underwent plain abdominal X-ray check 1 week after surgery to assess the status of the residual stones. Patients without or with residual stones $\leq 3 \mathrm{~mm}$, were defined as stone free. Notably, the study group lost significantly less intraoperative blood, had relatively low body temperature and a lower VAS score 1 day after surgery, a higher SFR 1 week after surgery, and substantially shorter postoperative indwelling catheter time than the control group patients $(p<0.05)$.

One patient in the study group $\left(\mathrm{T} 38.2^{\circ} \mathrm{C}\right)$, and 3 patients in the control group $\left(T \geq 38^{\circ} \mathrm{C}\right)$ developed postoperative fever. The postoperative temperature of 1 patient in the control group reached $39.1^{\circ} \mathrm{C}$. However, the body temperature of all patients returned to normal range after treatment, and no other serious complications such as septic shock occurred. Compared with the control group, the study group underwent relatively shorter operation time and hospitalization days were shorter, although sta-

Table I. Patient preoperative clinical data

\begin{tabular}{|c|c|c|c|}
\hline Variables & $\begin{array}{l}\text { Study } \\
(n=30)\end{array}$ & $\begin{array}{l}\text { Control } \\
(n=30)\end{array}$ & $P$-value \\
\hline Age [year] & $53.5 \pm 12.9$ & $55.7 \pm 10.8$ & 0.618 \\
\hline Sex: & & & 0.598 \\
\hline Male & $17(56.7)$ & 19 (63.3) & \\
\hline Female & $13(43.3)$ & $11(36.7)$ & \\
\hline Weight [kg] & $63.6 \pm 7.9$ & $65.8 \pm 7.8$ & 0.824 \\
\hline $\mathrm{BMI}$ & $25.2 \pm 3.2$ & $25.5 \pm 2.9$ & 0.592 \\
\hline First surgery: & & & 0.640 \\
\hline Yes & $28(93.3)$ & 27 (90.0) & \\
\hline No & $2(6.7)$ & $3(10.0)$ & \\
\hline Stone size $[\mathrm{mm}]$ & $18.2 \pm 5.3$ & $18.5 \pm 5.6$ & 0.853 \\
\hline Number of stones: & & & 0.787 \\
\hline Single & $19(63.3)$ & $20(66.7)$ & \\
\hline Multiple & $11(36.7)$ & $10(33.3)$ & \\
\hline $\begin{array}{l}\text { Urine leucocyte } \\
\text { negative: }\end{array}$ & & & 0.160 \\
\hline Yes & 1 & 2 & \\
\hline No & 29 & 28 & \\
\hline Urine culture: & & & 0.153 \\
\hline Positive & 0 & 2 & \\
\hline Negative & 30 & 28 & \\
\hline
\end{tabular}


Table II. Comparison of intraoperative and postoperative variables between the 2 groups

\begin{tabular}{|lccc|}
\hline Variables & $\begin{array}{c}\text { Study } \\
(n=30)\end{array}$ & $\begin{array}{c}\text { Control }(n \\
=30)\end{array}$ & P-value \\
\hline Operation time [min] & $57.3 \pm 10.6$ & $62.7 \pm 12$ & 0.132 \\
\hline Blood loss [ml] & $22.5 \pm 2.2$ & $27.3 \pm 3.1$ & 0.001 \\
\hline $\begin{array}{l}\text { Hospitalization time } \\
\text { days] }\end{array}$ & $2.3 \pm 0.9$ & $3.2 \pm 1.4$ & 0.171 \\
\hline Body temperature $\left[{ }^{\circ} \mathrm{C}\right]$ & $36.4 \pm 0.2$ & $36.7 \pm 0.3$ & 0.015 \\
\hline Postoperative fever $(n)$ & 1 & 3 & 0.301 \\
\hline Septic shock $(n)$ & 0 & 0 & - \\
\hline VAS pain score & $0.27 \pm 0.46$ & $0.61 \pm 0.73$ & 0.039 \\
\hline Stone-free rate $(\%)$ & 91.3 & 76.9 & 0.003 \\
\hline Catheter indwelling [days] & $1.6 \pm 0.84$ & $2.8 \pm 080$ & 0.015 \\
\hline
\end{tabular}

tistically insignificant $(p>0.05)$. These findings are summarized in Table II.

Regarding infection, there were no significant differences in preoperative blood routine, PCT, and CRP between the 2 groups $(p>0.05)$. Notably, the indexes for blood routine, PCT, and CRP in both groups were relatively high after surgery $(p<0.05)$. Furthermore, the control group displayed a higher infection index that the study group $(p<0.05)$. These results are summarized in Table III.

\section{Discussion}

Currently, there is a substantial increase in the adoption of minimally invasive procedures for surgical treatment for treatment of kidney stones. Although ESWL is a relatively non-invasive and economical procedure against kidney stones, its SFR is low. On the other hand, even though PNL has a higher SFR, it is associated with trauma and other adverse complications such as haemorrhage, pathogenic infections, and injury to adjacent organs [14]. Given the relatively high SFR and fewer trauma cases, FURL is gradually becoming the primary surgical choice against kidney stones. However, ureteral injury, haemorrhage, and other complications, particularly postoperative infection, have been reported following FURL. Research shows that the incidence of postoperative infection following FURL ranges between $1.7 \%$ and $18.8 \%$ [4]. Sepsis and even septic shock may occur in severe cases, with an incidence of approximately $3.4 \%$ and mortality as high as $66 \%$ [4]. This underlines the clinical significance of attention to postoperative infections.

Thus, we incorporated Soton ureteroscopy in FURL to explore whether this combination was superior to FURL alone in removing kidney stones regarding incidence and degree of infection. We found that a combination of Soton ureteroscope and FURL is superior to that of FURL alone. Soton ureteroscopy has been used in the clinical treatment of kidney stones in China only recently. Standard and operated ureteroscopy are the 2 types of Soton ureteroscopy. The Soton ureteroscope has a rigid outer sheath end connected to a negative-pressure suction device. During the operation, the standard ureteroscope was first inserted into the renal pelvis via the ureter using a rigid sheath to observe the stone. Then the

Table III. Comparison of infection index before and after surgery between the 2 groups

\begin{tabular}{|c|c|c|c|c|}
\hline Variables & & Preoperative & Postoperative & $P$-value \\
\hline \multirow[t]{3}{*}{ WBCs $\left[\times 10^{9}\right]$} & Study group & $7.0 \pm 0.8$ & $7.9 \pm 1.4$ & $<0.001$ \\
\hline & Control group & $6.8 \pm 1.2$ & $8.8 \pm 1.7$ & $<0.001$ \\
\hline & $P$-value & 0.138 & 0.015 & \\
\hline \multirow{3}{*}{$\begin{array}{l}\text { Percentage of } \\
\text { neutrophils (\%) }\end{array}$} & Study group & $61.0 \pm 7.9$ & $71.7 \pm 5.9$ & $<0.001$ \\
\hline & Control group & $60.3 \pm 7.7$ & $78.0 \pm 8.5$ & $<0.001$ \\
\hline & $P$-value & 0.730 & 0.002 & \\
\hline \multirow[t]{3}{*}{ CRP $[\mathrm{mg} / \mathrm{l}]$} & Study group & $1.60 \pm 0.65$ & $4.88 \pm 2.32$ & $<0.001$ \\
\hline & Control group & $1.72 \pm 0.71$ & $7.13 \pm 5.50$ & $<0.001$ \\
\hline & $P$-value & 0.453 & $<0.001$ & \\
\hline \multirow[t]{3}{*}{ PCT [ng/ml] } & Study group & $0.09 \pm 0.1$ & $0.58 \pm 0.35$ & $<0.001$ \\
\hline & Control group & $0.11 \pm 0.1$ & $1.05 \pm 0.90$ & $<0.001$ \\
\hline & $P$-value & 0.404 & $<0.001$ & \\
\hline
\end{tabular}


standard ureteroscope was removed and replaced with the operated ureteroscope. The renal stones were fragmented using a holmium laser and gravel powder, and perfused using negative-pressure suction. We used a combination of Soton and flexible ureteroscope for the study group. The lower pole renal and complicated stones were moved to the upper pole and renal pelvis using a stone basket aided by the flexible ureteroscope. Thereafter, lithotripsy was performed using the Soton ureteroscope. The negative-pressure suction device ensures a clear field of vision during the whole process while simultaneously maintaining renal pelvic pressure. Given that it allows suction of kidney stone fragments, it also facilitates lithotripsy. It also shortens the operation time, improves SFR, and reduces the risk of infection.

The use of ureteroscopic lithotripsy with negative-pressure suction, similar to Soton ureteroscope lithotripsy, has been reported in China and European countries. Lechevallier et al. used the irrigation/suction system for ureterorenolithotripsy and showed that it shortens the operation time and improves the SFR [15]. Du et al. demonstrated that applying the perfusion-suction platform in lithotripsy reduces the incidence of postoperative infection [16].

Huang et al. and Deng et al. reported that lithotripsy with a flexible suction ureteroscope increases SFR and reduces the incidence of postoperative complications $[9,17]$. However, there are no studies or discussions on the effect of negative-pressure suction systems on postoperative infection after lithotripsy.

Combining the previous results and the present study reports, our experience shows that the combination of Soton and flexible ureteroscope for the treatment of kidney stones can reduce the incidence and degree of postoperative infection, and its advantages are as follows.

1. Clear vision. During lithotripsy using a Soton ureteroscope, bubbles, blood clots, gravel particles, and stone powder can be actively sucked out by the negative-pressure suction device. This significantly reduces interference and improves visual clarity. This also reduces accidental holmium laser damage to the kidney during lithotripsy and the probability of bacteria inside the stone entering the blood. Moreover, our study results show that the amount of intraoperative blood loss in the study group was significantly less than the control group. Thus, the result indirectly confirms that the degree of renal injury was less when the Soton ureteroscope was used during the operation.

2. Good control of renal pelvic pressure. As the intraoperative calculi are shattered by laser, infectious materials and metabolites can enter the bloodstream through the locally injured mucosa, leading to infection and even the occurrence of SIRS [18]. Studies have shown that when the renal pelvis perfusion pressure is exceptionally high, it can lead to reperfusion, as well as systemic infection leading to postoperative fever, infection, and even sepsis [19]. However, with Soton ureteroscope lithotripsy and a negative-pressure suction device, fluid could be actively drained while minimizing perfusion fluid absorption and maintaining renal pelvis pressure, which invariably reduces infection risk.

3. Shorter operation time. Longer operation time increases the risk of postoperative infection. The stone basket used in traditional flexible URS is time consuming [4]. However, during Soton ureteroscopy lithotripsy and simultaneous gravel removal, the repeated use of a stone basket is avoided, and the flushing of stones with high-pressure perfusion is reduced. It can also clear stones faster, thus improving efficiency. Also, stone movement can increase the operation time; however, Soton ureteroscopy in combination with negative pressure during lithotripsy reduces stone displacement, prevents stone movement, and ensures continued lithotripsy. This effectively accelerates the entire process, reducing the operation time.

In this study, the Soton ureteroscope was successfully inserted into the renal pelvis in all study cases, but in 21 cases, due to some calculi in calyx that could not be directly treated by Soton ureteroscope, the flexible ureteroscope was replaced intraoperatively to adjust the position of calculi. Even though the difference in the operation time between the two groups of patients was not statistically significant, the average operation time of the study group was relatively short and thus clinically significant. We believe there was no significant difference between the 2 groups because of the time it took to replace the 2 ureteroscopes in the study group, i.e. about $5 \mathrm{~min}$ on average. Namely, the actual lithotripsy time was shorter than recorded in the study group.

In this study, the main purpose of the postoperative indwelling catheter was to prevent the backflow 
of urine along the ureteral stent and bacteria into the blood, resulting in early postoperative infection. Theoretically, Soton ureteroscopic lithotripsy causes less damage to the kidney and less possibility of bacteria entering the blood. In order to the rapidly recovery and discharge patients as soon as possible, the indwelling time of catheter can be appropriately shortened. The results showed that it was safe to shorten the indwelling time of the catheter in the study group.

Our study results showed no significant difference in preoperative infection indicators between the 2 groups $(p>0.05)$. However, postoperative blood routine inflammation indicators such as WBCs and percentages of neutrophils were significantly higher in the control than in the study group. Infection indicators such as CRP and PCT were also significantly higher in the control group.

Also, only 1 patient in the study group developed fever $\left(38^{\circ} \mathrm{C}\right)$ but had a negative blood culture. So, the possibility of absorption fever after surgery was considered. However, 2 patients in the control group developed fever with body temperature greater than $38^{\circ} \mathrm{C}$, while 1 had a maximum body temperature of $39.1^{\circ} \mathrm{C}$. Overall, compared with FURL alone, there were significantly fewer postoperative infections following Soton and flexible ureteroscopy lithotripsy.

This article has several limitations. The present research is a single-centre study with a relatively small sample size. Also, we did not monitor renal pelvic pressure during lithotripsy. Despite these limitations, this study showed the value of the application of the Soton ureteroscope in flexible ureteroscopy lithotripsy. In research to follow, we shall perform utilize larger multicentre data to validate the present study results.

\section{Conclusions}

The study showed that the Soton ureteroscope with FURL is a safe and effective minimally invasive surgical method used to treat renal calculi. Also, postoperative infection after treatment is significantly less than flexible ureteroscopy lithotripsy alone. Thus, the application of the Soton ureteroscope has the advantage of preventing infection after flexible ureteroscopy lithotripsy.

\section{Conflict of interest}

The authors declare no conflict of interest.

\section{Reference}

1. Scales CD Jr. Practice patterns in the management of urinary lithiasis. Curr Urol Rep 2013; 14: 154-7.

2. Türk C, Petřík A, Sarica K, et al. EAU Guidelines on Interventional Treatment for Urolithiasis. Eur Urol 2016; 69: 475-82.

3. Baş O, Tuygun C, Dede O, et al. Factors affecting complication rates of retrograde flexible ureterorenoscopy: analysis of 1571 procedures-a single-center experience. World J Urol 2017; 35: 819-26.

4. Fan S, Gong B, Hao Z, et al. Risk factors of infectious complications following flexible ureteroscope with a holmium laser: a retrospective study. Int J Clin Exp Med 2015; 8: 11252-9.

5. Yu W, Cheng F, Zhang X, et al. Retrograde ureteroscopic treatment for upper ureteral stones: a 5-year retrospective study. J Endourol 2010; 24: 1753-7.

6. Campobasso D, Grande M, Ferretti S, et al. Subcapsular renal hematoma after retrograde ureterorenoscopic lithotripsy: our experience. Minerva Urol Nefrol 2018; 70: 617-23.

7. Zhong W, Leto G, Wang L, et al. Systemic inflammatory response syndrome after flexible ureteroscopic lithotripsy: a study of risk factors. J Endourol 2015; 29: 25-8.

8. Cindolo L, Castellan P, Scoffone CM, et al. Mortality and flexible ureteroscopy: analysis of six cases. World J Urol 2016; 34: 305-10.

9. Huang J, Xie D, Xiong R, et al. The application of suctioning flexible ureteroscopy with intelligent pressure control in treating upper urinary tract calculi on patients with a solitary kidney. Urology 2018; 111: 44-7.

10. Meisner M, Tschaikowsky K, Palmaers T, et al. Comparison of procalcitonin (PCT) and C-reactive protein (CRP) plasma concentrations at different SOFA scores during the course of sepsis and MODS. Crit Care 1999; 3: 45-50.

11. Castelli GP, Pognani C, Meisner M, et al. Procalcitonin and C-reactive protein during systemic inflammatory response syndrome, sepsis and organ dysfunction. Crit Care 2004; 8: R234-42.

12. Masajtis-Zagajewska A, Nowicki M. New markers of urinary tract infection. Clin Chim Acta 2017; 471: 286-91.

13. Jin L, Yang B, Zhou Z, et al. Comparative efficacy on flexible ureteroscopy lithotripsy and miniaturized percutaneous nephrolithotomy for the treatment of medium-sized lower-pole renal calculi. J Endourol 2019; 33: 914-9.

14. Chen Y, Wen Y, Yu Q, et al. Percutaneous nephrolithotomy versus flexible ureteroscopic lithotripsy in the treatment of upper urinary tract stones: a meta-analysis comparing clinical efficacy and safety. BMC Urol 2020; 20: 109.

15. Lechevallier E, Luciani M, Nahon O, et al. Transurethral ureterorenolithotripsy using new automated irrigation/suction system controlling pressure and flow compared with standard irrigation: a randomized pilot study. J Endourol 2003; 17: 97-101.

16. Du C, Song L, Wu X, et al. A study on the clinical application of a patented perfusion and suctioning platform and ureteral access sheath in the treatment of large ureteral stones below L4 level. Int Urol Nephrol 2019; 51: 207-13. 
17. Deng X, Song L, Xie D, et al. A novel flexible ureteroscopy with intelligent control of renal pelvic pressure: an initial experience of 93 cases. J Endourol 2016; 30: 1067-72.

18. Erdil T, Bostanci Y, Ozden E, et al. Risk factors for systemic in flammatory response syndrome following percutaneous nephrolithotomy. Urolithiasis 2013; 41: 395-401.

19. Kreydin El, Eisner BH. Risk factors for sepsis after percutaneous renal stone surgery. Nat Rev Urol 2013; 10: 598-605.

Received: 15.12.2020, accepted: 13.05.2021. 